primary care by training clinical officers (Jenkins et al, 2010b, 2013). A stepped-care strategy also applied in settings with higher densities of specialised providers.

The findings from our survey point to a significant role played by psychiatric nurses in the private sector - through private out-patient practice, in-patient locum work and with NGOs. One nurse noted that the Ministry of Health has a policy prohibiting simultaneous work in public and private facilities, out of concern that private work compromises public work: 'If people work two jobs, they do it quietly.' Nonetheless, $14 \%$ of nurses reported doing locum work. A further $10 \%$ had managed a private practice and a quarter of their patients came seeking mental health services. Clinics run by psychiatric nurses tend to operate outside Nairobi, as the competition from private psychiatrists is high in the capital. The participation rate of psychiatric nurses in private practice was found to be lower than in other types of nursing (Barnes et al, 2010), though still significant.

A limitation of this study is the potential for sampling bias, as the sample represents only those attending the annual general conference on mental health. Those with psychiatric nursing qualifications who are no longer practising mental health would be less inclined to attend a mental health meeting. It is also possible that those in private practice would be less likely to attend the meeting, since there is a separate chapter of the NNAK for nurses in private practice. If this is the case, then our estimate of the shortfall of psychiatric nurses is conservative, as is our estimate of their participation in private practice. In addition, the questionnaire did not address wider intersectoral roles held by psychiatric nurses, including supervising primary care.

In conclusion, it appears that psychiatric nurses are migrating internally to nursing positions in other areas of healthcare, aggravating the existing 'brain drain' for mental health.

\section{References}

Barnes, J., O'Hanlon, B., Feeley, III, F., et al (2010) Private Health Sector Assessment in Kenya. World Bank.

Chetty, D. \& Hoque, M. (2013) Effectiveness of a nurse facilitated cognitive group intervention among mild to moderately depressed women in KwaZulu-Natal, South Africa. African Journal of Psychiatry, 16, 29-34.

Jenkins, R., Kydd, R., Mullen, P., et al (2010a) International migration of doctors, and its impact on availability of psychiatrists in low and middle income countries. PLoS One, 5(2), e9049.

Jenkins, R., Kiima, D., Njenga, F., et al (2010b) Integration of mental health into primary care in Kenya. World Psychiatry, 9, 118-120.

Jenkins, R., Othieno, C., Okeyo, S., et al (2013) Short structured general mental health in service training programme in Kenya improves patient health and social outcomes but not detection of mental health problems - a pragmatic cluster randomised controlled trial. International Journal of Mental Health Systems, 7, 25.

Kakuma, R., Minas, H., van Ginneken, N., et al (2011) Human resources for mental health care: current situation and strategies for action. Lancet, 378, 1654-1663.

Kiima, D. \& Jenkins, R. (2010) Mental health policy in Kenya - an integrated approach to scaling up equitable care for poor populations. International Journal of Mental Health Systems, 4(19), 1-8.

Kohn, R., Saxena, S., Levav, I., et al (2004) The treatment gap in mental health care. Bulletin of the World Health Organization, 82, 858-866.

Padmanathan, P. \& Newell, J. N. (2012) Retention factors affecting migrant psychiatrists from low- and middle-income countries. International Psychiatry, 9, 13-15.

Rakuom, C. (2010) Nursing Human Resources in Kenya: Case Study. International Centre for Human Resources in Nursing \& Florence Nightingale International Foundation.

World Health Organization (2011) Mental Health Atlas 2011. WHO.

SPECIAL PAPER

Professor of International Mental Health and Wellcome Trust Senior Research Fellow in Clinica Science; The Centre for Global Mental Health, London School of Hygiene and Tropical Medicine; Sangath; and Centre for Chronic Conditions and Injuries, Public Health Foundation of India

The research described in this article has been supported by the Wellcome Trust (V.P. is supported by a Wellcome Trust Senior Research Fellowship in wship in Clinical Science); the Nationa Institute for Mental Health; Autism Speaks; the Jamsetji Tata Trust; the WHO; the MacArthur Foundation; and UNFPA. The author is a founder of Sangath d a member of its Managing Committee.

\title{
SUNDAR: mental health for all by all
}

\author{
Vikram Patel
}

This article describes the rationale and principles for the SUNDAR approach adopted by Sangath, an Indian non-governmental organisation: to use lay people, with appropriate training and supervision, to deliver psychosocial interventions for a range of mental health conditions. This approach has been evaluated in a number of randomised controlled trials and is now being scaled up. At the core of this innovation is revisiting the questions of what constitutes mental healthcare, who provides mental healthcare and where mental healthcare is provided. In doing so, SUNDAR offers a vision for a mental healthcare system which is empowering, inclusive, equitable and effective.

\section{The rationale}

Even by the most conservative prevalence estimates, about 50 million people are affected by mental health problems in India. In contrast to this high burden, the country has only approximately 5000 mental health professionals. It is obvious then that the strategies to address mental healthcare need to rely on alternative human resources if the country is to meet more than a tiny fraction of the needs of people affected by mental health problems. It is in this context that Sangath (http://www.sangath. com), an Indian non-governmental organisation (NGO) headquartered in the state of Goa and now working in several states around the country, began shaping its approach to using lay people to 
deliver evidence-based psychosocial interventions for mental health problems. Inspired by similar approaches to 'task-sharing' interventions with lay and community-based workers in other areas of healthcare in India (for example, India's National Rural Health Mission contracts nearly a million such workers to deliver a range of maternal and child health interventions), Sangath adopted an approach which has now been replicated for use across a diverse range of mental health conditions.

\section{The SUNDAR approach}

Sangath's approach is characterised by several principles:

- designing interventions based on global evidence of effectiveness and local evidence of cultural acceptability

- systematically testing intervention delivery to ensure the feasibility of its use by lay health workers (who are referred to as 'counsellors') and its acceptability by patients and families

- involving diverse stakeholders, in particular people affected by the target mental health problems, in shaping the content and delivery of the intervention

- embedding the intervention in established healthcare platforms, most commonly those run by the government but also the private sector, which is widely utilised in India, to ensure scalability

- evaluating the effectiveness and costeffectiveness of the intervention in randomised controlled trials in partnership with leading research institutions, notably the Centre for Global Mental Health in London (http://www. centreforglobalmentalhealth.org)

- disseminating the findings in a variety of ways, ranging from scientific papers to audiovideo media (see for example https://vimeo. com/67216615)

- working closely with federal and state ministries of health to scale up the innovations.

Several lessons have emerged from these experiences, which have been coined with the acronym SUNDAR (which means 'attractive' in the Hindi language).

- First, we should Simplify the messages we use to convey mental health issues, for example replacing psychiatric labels which can cause shame or misunderstanding with those which are contextually appropriate and widely understood.

- Second, we should Unpack our interventions into components which are easier to deliver and incorporate culturally sensitive strategies.

- Third, these unpacked interventions should be Delivered as close as possible to people's homes, typically their actual homes or the nearest primary healthcare centre or community facility.
- Fourth, we should recruit and train Available human resources from the local communities to deliver these interventions. This often refers to lay counsellors, but could also include parents and teachers in the case of childhood disorders.

- And finally, we should judiciously Re-allocate the scarce and expensive resource of mental health professionals to design and oversee mental healthcare programmes, and train, supervise and support community health agents.

This approach is built around a collaborative care framework, the most evidence-based delivery model for integrating mental health into routine healthcare platforms (Patel et al, 2013a), with four key human resources: the front-line lay counsellor; the person with a mental health problem and the family; the primary or general healthcare physician; and the mental health professional. SUNDAR is attractive not only because it enhances access to care using available human resources in an efficient way, but also because it empowers ordinary people to provide mental healthcare for others and, in so doing, promotes their own wellbeing (Shinde et al, 2013).

\section{The evidence}

Based on this approach, Sangath has completed research involving the systematic development of interventions and subsequent randomised controlled trials of interventions for three mental health problems - dementia, schizophrenia and common mental disorders - all of which have shown significant benefits in terms of clinical or social outcomes. The dementia trial (the Home Care Trial) (Dias et $a l, 2008)$ was the first such study from a low-income country and won Alzheimer Disease International's international prize for psychosocial interventions in 2010. The common mental disorders trial (the MANAS Trial) was the largest trial in psychiatry in low- and middle-income countries and the first to demonstrate the cost-effectiveness of task-sharing for mental healthcare (Patel et al, 2011; Buttorff et al, 2012). The schizophrenia trial demonstrated modest benefits in reducing disability levels in people with chronic illness (Chatterjee et al, 2014).

Six projects following the SUNDAR approach are currently in progress, for: the treatment of alcohol use disorders in primary care; the treatment of severe depression in primary care; the treatment of maternal depression in community settings by peers (i.e. other mothers); parent-mediated interventions for autism; school mental health promotion for young people; and the prevention of depression in late life.

\section{The impact}

This evidence has been used to scale up mental healthcare in rural communities in one of the poorest regions of the country through VISHRAM (Vidarbha Stress and Health Program), a partnership between Sangath, social development NGOs, the Ministry of Health and psychiatrists. 
Promisingly, the new National Mental Health Programme of the Ministry of Health (government of India), which finances the country's District Mental Health Programme, citing this evidence, has mandated the establishment of a new cadre of community mental health worker attached to primary healthcare centres throughout this vast country. This evidence, consistent with similar evidence from other low- and middle-income countries on the effectiveness of psychosocial interventions delivered by lay health workers (van Ginneken et al, 2013), has led to this approach being one of the key recommendations of the World Innovation Summit in Health, in its report on mental health (Patel $e t$ al, 2013b). Examples of such innovations can be found on the recently launched Mental Health Innovations Network (http://mhinnovation.net/innovation/).

However, this research also points to some of the limits of this approach. For example, the evidence is restricted to a few mental health conditions, has not been evaluated for the critically important procedure of diagnosis and has not yet been scaled up significantly in any country. Thus, it is reassuring that these gaps in knowledge have led to a revolution in the field of global mental health research, with task-sharing among the leading research priorities in the Grand Challenges for Global Mental Health (Collins et al, 2011), which has leveraged more than US $\$ 50$ million in the past 2 years to support more research and capacity building in this area.

There are a number of NGOs working in low-income countries to build skills among community-based workers to deliver psychosocial treatments for mental health problems, but few that are using robust, peer-reviewed scientific methods to evaluate the effect of these approaches and working closely with ministries of health to scale up these innovations. Sangath stands out as a rare example of an innovator committed to community empowerment, science and scaling up in low-resource settings. The impact of this evidence on the recognition of Sangath as one of India's leading mental health research institutions has been significant and a range of professionals from around the world participate in its annual 2-week Leadership in Mental Health course, held in November in Goa, to learn about these models of care.

\section{The relevance for global mental health}

The SUNDAR innovation of task-sharing of mental healthcare with lay people has enormous potential for low- and middle-income countries. While it would come as no surprise to learn that there are astonishingly large gaps in access to evidencebased care in these countries, the real puzzle is that large proportions of people do not access such care even in high-income countries. There are many explanations for this observation. At the heart of them all is the remoteness of mental healthcare from the communities it serves: the interventions are heavily medicalised; they do not engage sufficiently with harnessing personal and community resources; they are delivered in highly specialised and expensive settings; and they use language and concepts which alienate ordinary people. In all these respects, the SUNDAR approach may be instructive to rethinking mental healthcare globally.

At the core of this innovation is revisiting the questions of what constitutes mental healthcare, who provides mental healthcare and where mental healthcare is provided. SUNDAR uses appropriately trained and supervised lay workers, working in settings and at times convenient to patients (even in patients' homes and outside regular working hours). It offers a range of contextually appropriate interventions tailored to the needs of the individual and using familiar labels and concepts. SUNDAR is an approach with relevance to rethinking mental healthcare in all countries. By acting on the axiom that mental health is too important to be left to mental health professionals alone, SUNDAR seeks to achieve a paradigm shift by reframing so-called 'under-resourced' communities as 'richly resourced', for there is surely no community on earth which is not richly endowed with human beings who are capable of caring for those with mental health problems.

\section{References}

Buttorff, C., Hock, R. S., Weiss, H. A., et al (2012) Economic evaluation of a task-shifting intervention for common mental disorders in India. Bulletin of the World Health Organization, 90 813-821.

Chatterjee, S., Naik, S., John, S., et al (2014) Effectiveness and costeffectiveness of a community based intervention for people with schizophrenia and their caregivers in India: the COPSI randomised controlled trial. Lancet, 383, 1385-1394.

Collins, P. Y., Patel, V., Joestl, S. S., et al (2011) Grand challenges in global mental health. Nature, 475, 27-30

Dias, A., Dewey, M. E., D'Souza, J., et al (2008) The effectiveness of a home care program for supporting caregivers of persons with dementia in developing countries: a randomised controlled trial from Goa, India. PLOS ONE, 3(6), e2333.

Patel, V., Weiss, H. A., Chowdhary, N., et al (2011) Lay health worker led intervention for depressive and anxiety disorders in India: impact on clinical and disability outcomes over 12 months. British Journal of Psychiatry, 199, 459-466.

Patel, V., Belkin, G. S., Chockalingam, A., et al (2013a) Grand challenges: integrating mental health services into priority health care platforms. PLoS Medicine, 10(5), e1001448.

Patel, V. \& Saxena, S., with De Silva M. \& Samele, C. (2013b) Transforming Lives, Enhancing Communities: Innovations in Mental Health. World Innovation Summit for Health. Available at http:// www.wish-qatar.org/app/media/381 (accessed December 2014)

Shinde, S., Andrew, G., Bangash, O., et al (2013) The impact of a lay counselor led collaborative care intervention for common mental disorders in public and private primary care: a qualitative evaluation nested in the MANAS trial in Goa, India. Social Science and Medicine, $88,48-55$.

van Ginneken, N., Tharyan, P. Lewin, S., et al (2013) Non-specialist health worker interventions for the care of mental, neurological and substance-abuse disorders in low- and middle-income countries. Cochrane Database of Systematic Reviews, 11, CD009149. 